\title{
Magnetic resonance imaging indicator of the causes of optic neuropathy in IgG4- related ophthalmic disease
}

\author{
Jing Li ${ }^{2 \dagger}$, Yan Zhang ${ }^{3 \dagger}$, Hang Zhou ${ }^{4}$, Lei Wang ${ }^{5}$, Zhenchang Wang ${ }^{2}$ and Hongyang $\mathrm{Li}^{1^{*}}$ (D)
}

\begin{abstract}
Background: The following study investigates the involvement of optic neuropathy in IgG4-related ophthalmic diseases (IgG4-ROD) based on the magnetic resonance imaging (MRI) data, and different imaging features of IgG4ROD related optic neuropathy related to other orbital diseases.

Methods: This retrospective study included 225 patients with IgG4-RD admitted at two ophthalmology centers between January 2014 and December 2017. Twenty-six patients had both pre-therapeutic orbital MRI and optic never injury. The causes of optic neuropathy were analyzed, and the special sign in MRI to diagnose IgG4-ROD was also evaluated.

Results: Twelve cases had inflammation of the optic nerve sheath, while 14 cases had compression due to extraocular muscles and pseudo tumor masses. Two cases had hypertrophic cranial pachymeningitis, while one case had hypophysis involving optic chiasma.

Conclusion: The most common causes of optic nerve injury in IgG-4 ROD are inflammation of optic nerve sheath, compression of extraocular muscles, pseudo tumor mass and hypertrophic cranial pachymeningitis, and hypophysis involving optic chiasma.
\end{abstract}

Keywords: IgG4-related ophthalmic disease, Optic neuropathy, Magnetic resonance imaging, Cause of disease

\section{Background}

IgG4-related disease (IgG4-RD) is a chronic inflammatory disorder characterized by IgG4-positive lymphoplasmacytic infiltrative lesions and elevated serum IgG4 levels $[1,2]$. Target organs that are most commonly affected by IgG4-RD are the orbits, salivary and lacrimal glands, lungs, kidneys, prostate, aorta and retroperitoneum, lymph nodes, biliary tree and as visceral inflammatory pseudo tumours. In the USA, 23\% of all of IgG4$\mathrm{RD}$ cases, the disease manifests in the eye or orbit. Furthermore, lacrimal gland swelling was found in 4 to $34 \%$ IgG4-RD cases in Japanese reviews.

Eyes are often the first most frequent site associated with IgG4-RD [3]. A number of orbital inflammatory cases,

\footnotetext{
*Correspondence: faraway_sweet@163.com

${ }^{\dagger}$ Jing Li and Yan Zhang contributed equally.

'Department of Ophthalmology, Beijing Friendship Hospital, Capital Medical University, No. 95 Yong'an Road, Xicheng District, Beijing 100050, People's Republic of China

Full list of author information is available at the end of the article
}

formerly considered as other idiopathic lesions, are now regarded as IgG4-related orbital diseases (IgG4-ROD) [4]. Radiology image is important to distinguish IgG4-ROD from other orbital diseases. IgG4-ROD usually presents as dacryoadenitis, myositis, or inflammation of infraorbital fat. In addition, some researches have indicated that the presence of infraorbital nerve enlargement (IONE) is considered as a key sign of IgG4-ROD on an MRI [5].

Optic neuropathy may be involved in IgG4-ROD, and it can lead to severe injuries. However, this type of disease is more likely misdiagnosed as other orbital inflammatory diseases, such as optic neuritis, Graves' disease, inflammatory pseudotumor, lymphoma, sarcoidosis and so on [6]. IgG4-ROD is quite different from these diseases with reference to treatment and prognosis [3]. Therefore, its early diagnosis is very important. Over the recent years, much knowledge has been gained about the clinical manifestations of IgG4-ROD [7]; yet, most of studies were small case 
series, and only few explored the imaging manifestations of IgG4-ROD related optic neuropathy. Clinical data have suggested that decreased visual acuity and defective visual field are the first symptoms to appear. Nevertheless, most of the existing studies have focused on dacryoadenitis, myositis and branch of trigeminal neuropathy $[8,9]$. In addition, there is insufficient information on radiological features of optic neuropathy with IgG4ROD, and causes of optic neuropathy in IgG4-ROD.

Hence, the purpose of this study was to investigate the radiological features of optic neuropathy from IgG4-ROD in Chinese patients. Furthermore, we suggest a radiological diagnostic criterion for optic neuropathy cases diagnosed as IgG4-ROD.

\section{Materials and methods}

\section{Patients}

This retrospective study included 225 patients with IgG4-RD admitted at two ophthalmology centers between January 2014 and December 2017. Ocular manifestations were the first symptoms of IgG4-RD. IgG4-RD was diagnosed based on the Japanese Comprehensive Diagnostic Criteria for IgG4-RD [10]: (1) clinical examination showing characteristic diffuse/ localized swelling or masses in single or multiple organs; (2) hematological examination showing elevated serum IgG4 concentrations ( $\geq 135 \mathrm{mg} / \mathrm{dL}$ ); (3) histopathological examination showing marked lymphocyte and plasma cell infiltration and fibrosis and Infiltration of IgG4+ plasma cells: ratio of IgG4+ to IgG+ cells $>40 \%$ and $>10$ IgG4+ plasma cells/high power field (HPF). Based on these criteria, the patients were diagnosed with IgG4-RD (definite: $(1)+(2)+(3)$; probable: $(1)+(3)$; possible: $(1)+(2))$.

The exclusion criteria were: ocular manifestations were not the first symptoms; pretherapeutic MRI was insufficient for an adequate interpretation; absence of IgG4 testing during the pathology examination; thyroid-related eye diseases and lymphoma and other orbital- related diseases; ocular exam that was insufficient for analysis; patients without visual loss (best corrected visual acuity $<1.0$ or visual field defect) or optic neuropathy (optic atrophy or papilledema).

A flowchart illustrating the selection of patients is shown in Fig. 1. A total of 199 patients did not meet the inclusion criteria. Finally, 26 patients were included in the study.

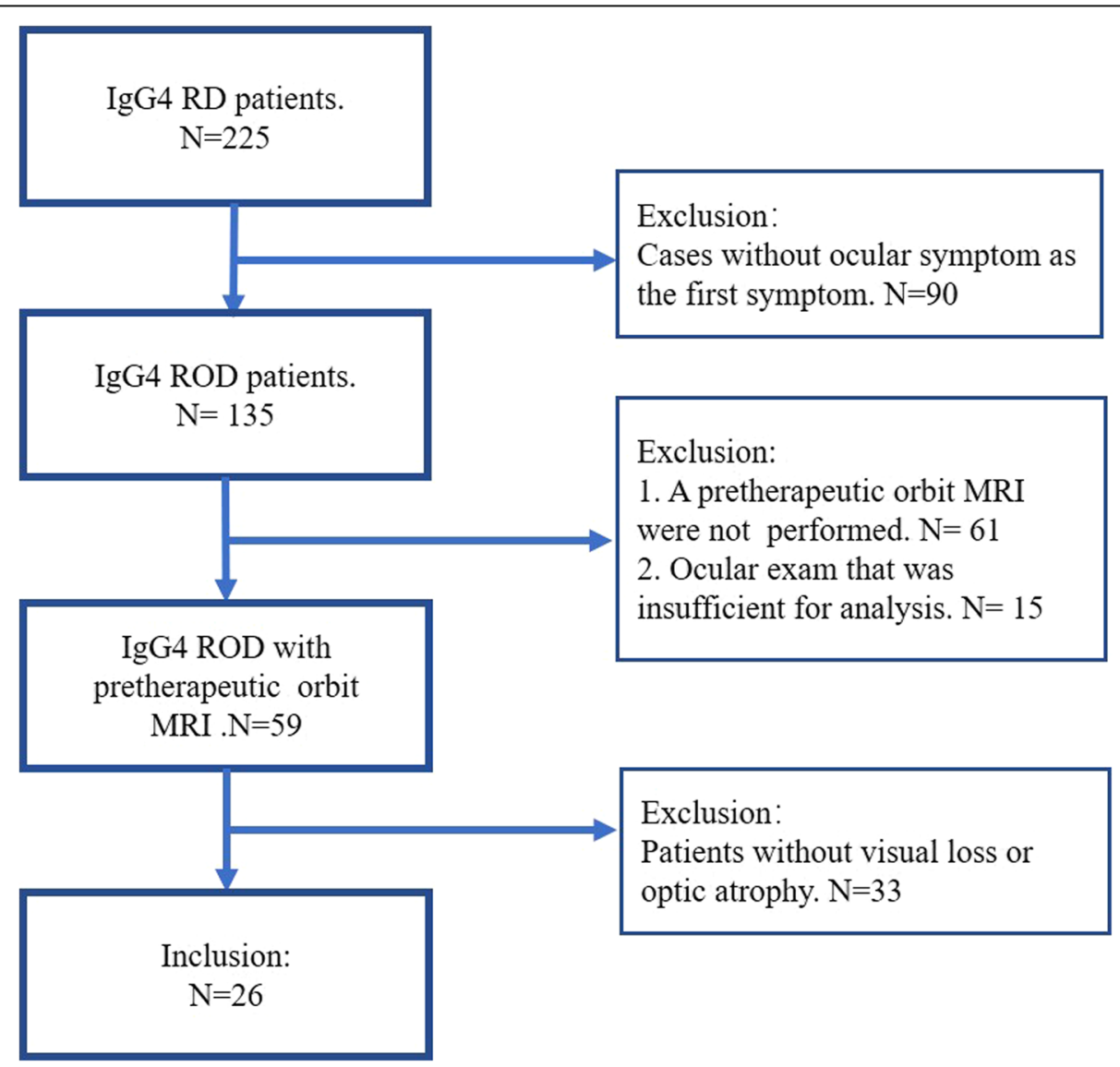

Fig. 1 A total of 26 patients met the inclusion criteria 
Review of optic examination

Ophthalmic examinations included slit lamp examination and pupillary reactions in unilateral or bilateral. Visual acuity was examined by the standard table of vision logarithms at $5 \mathrm{~m}$. Those unable to read any letters at the distance of 1 meter were further examined by finger counts, hand movements, or perceiving light. Visual field was performed by Humphrey field Analyzer (30-2 SITA, Humphrey 750i, Zeiss, Germany). Digital fundus photographs were obtained using nonmydriatic fundus photography. Binocular digital photographs were taken using a $45^{\circ}$ high-resolution fundus camera (Kowa, Tokyo, Japan); and retinal fundus images were centered on the optic disc.

\section{Review of MR imaging}

The MRI of the orbit was evaluated using MRI-3.0 T (TW1WSPEED HDXT, GE, USA). Scanning sequences and parameters were the following: coronal T1-weighted Fast spin-echo $(\mathrm{TR}=660 \mathrm{~ms} ; \mathrm{TE}=11.1 \mathrm{~ms}$, matrix size $=$ $256 \times 256 \mathrm{~mm}, \mathrm{FOV}=18 \times 18 \mathrm{~cm}$, slice thickness $=3.0 \mathrm{~mm}$ ); Gd-DTPA $0.1 \mathrm{mmol} / \mathrm{kg}$ was used as contrast agent for enhanced MRI, and was combined with fat suppression scan technique. The MR imaging of patients was evaluated by two experienced neuroradiologists (12 and 8 years of imaging experience). From the coronal imaging plane, each extraocular muscle was measured in 2 dimensions (millimeters): maximum diameter and maximum short axis $[11,12]$.

Table 1 Demographic and clinical characteristics

\begin{tabular}{ll}
\hline Number of patients ( $\mathrm{n})$ & 26 \\
\hline Age (years) & $51-78$ \\
Sex (male:female) & $12: 14$ \\
Bilaterality (n) & 26 \\
Extrapohthalmic involvement (n) & 21 \\
steroid-sensitive patients (n) & 23 \\
Auto-antibody (n) & 5 \\
ANA & 4 \\
SSA/SSB & 0 \\
thyroid autoantibodies & 0 \\
RF & 1 \\
HLA & 0 \\
Others & 0 \\
Vision loss (n) & 26 \\
Vision field defect (n) & 26 \\
optic atrophy (n) & 10 \\
papilledema (n) & 5 \\
\hline
\end{tabular}

\section{Results}

Patient demographics

A total of 26 patients met the inclusion criteria (Table 1) . The male: female ratio was 12:14, and the age ranged from 51 to 78 years. There were 23 patients with extraophthalmic involvement, including parotid gland, submandibular gland, sinusitis, pancreatitis, lymph nodes enlargement, nephrosis, cholangitis and pneumonia. All patients had bilateral involvement.

\section{Clinical features of optic nerve injury}

The manifestations of optic nerve injury were visual impairment, visual field detection, papilledema or optic atrophy. The most common manifestations of optic nerve sheath inflammation were mild visual impairment, mild papilledema and peripheral visual field defect (Fig. 2).

\section{MRI indicator of the causes for optic neuropathy in IgG4- ROD}

Compression was observed in more than half of patients with optic neuropathy, including hypophysis involving the optic chiasm (1 case, Fig. 3). MRI showed diffuse enlargement of the pituitary and infundibulum, resulting in the compression of optic chiasm. Two cases with hypertrophic cranial pachymeningitis (Fig. 4), and 14 cases were compressed by pseudotumor masses and extraocular muscles (EOMs) hypertrophy (Fig. 5), MRI showed hyperintense linear dura thickening; thickened dura mater was markedly enhanced after contrast media, compressing the optic nerve in the orbit apex, and pseudotumor mass circles of optic nerve. In 12 patients with optic neuropathy who suffered from optic nerve sheath inflammation (Fig. 6), MRI showed that optic neuritis was enhanced and was characterized by diffuse thickening of the optic nerve sheath, and blurred margin. Furthermore, optic nerve sheath inflammation often appeared with infraorbital nerve enlargement (Fig. 7).

\section{Discussion}

IgG4-RD is characterized by elevated serum IgG4 levels and IgG4-positive lymphoplasmacytic infiltrative lesions [1, 2] and it involves multiple organs, including ocular structures [13, 14]. Previous studies, investigating IgG4-related ophthalmic diseases, have been mainly focused on examining ocular adnexa, dacryoadenitis, myositis and branch of trigeminal neuropathy lesions. In contrast, optic neuropathy is a frequent cause of vision loss encountered by ophthalmologist, which often leads to more severe injuries compered to ocular adnexa lesions. Thus, real-team imaging methods are an important tool for identifying the pathogenesis of optic neuropathy.

In the current study, we used MRI to determine the causes of optic neuropathy development, such as 

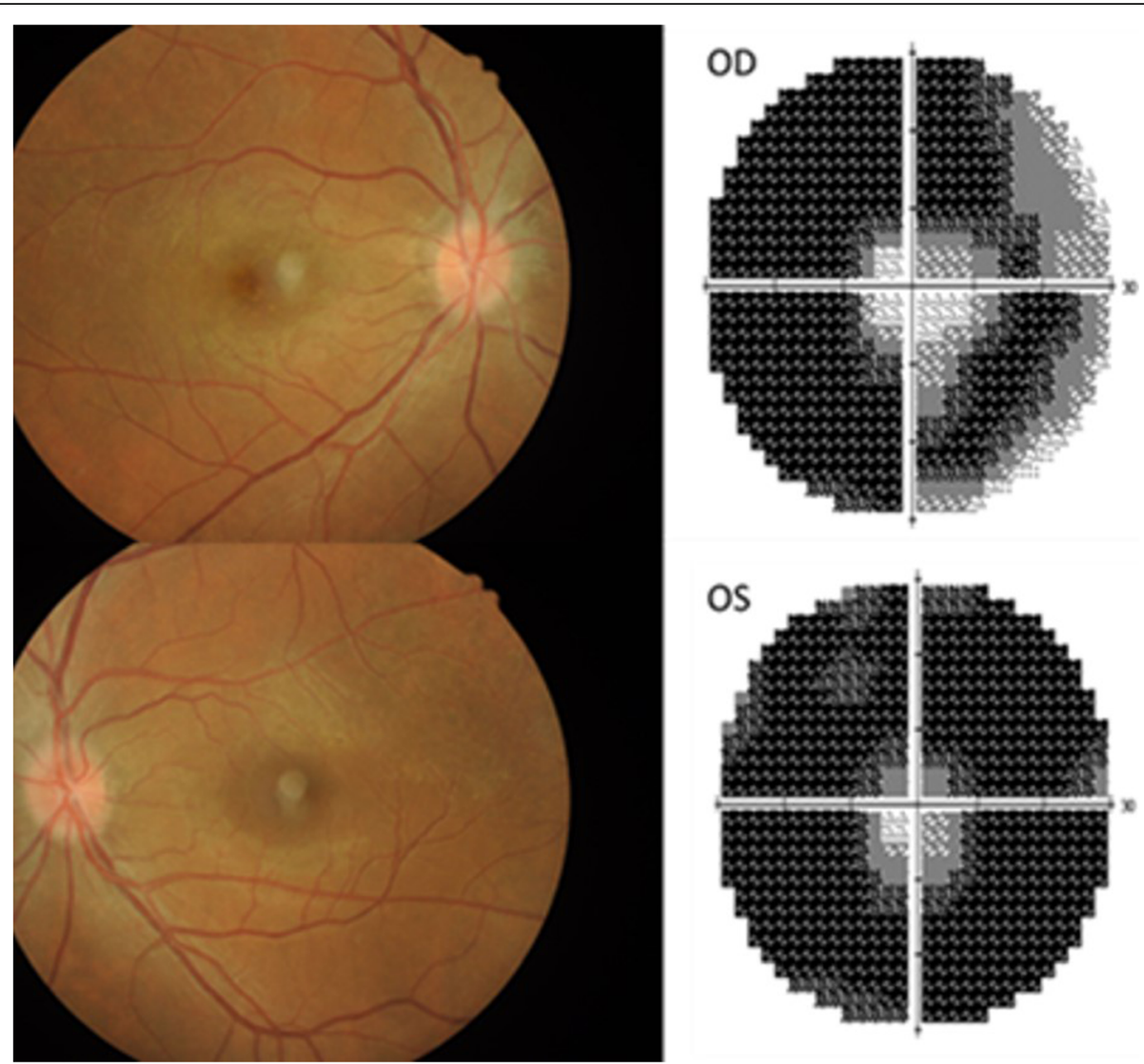

Fig. 2 The main performance of optic nerve injury. A patient with bilateral injury from IgG4-ROD. Bilateral papilledema was performed using fundus camera and peripheral visual field defect through Humphrey field Analyzer



Fig. 3 lgG4-RD with hypophysis involving optic chiasma. coronal (a) and Sagittal (b) contrast enhanced T1-weighted MRI showed diffuse enlargement of the pituitary and infundibulum, resulting in the compression of optic chiasm. White arrows show the opposition of optic chiasm 




Fig. 4 Compression of optic nerve as hypertrophic cranial pachymeningitis. Coronal contrast enhanced T1-weighted MRI (a) showing hyperintense linear dura thickening (arrow heads). Sagittal contrast enhanced T1-weighted MRI (b) showing the thickened dura mater was markedly enhanced after contrast media, and compressed the optic nerve in the orbit apex (white arrow)

hypophysis, extraocular muscle enlargement, hypertrophic cranial pachymeningitis, compressed by pseudotumor masses and optic nerve sheath inflammation. Currently, the most commonly used imaging modalities for IgG4-ROD are computed tomography (CT) and MRI [15-17]. In the current study, all the patients underwent MRI, since CT imaging of optic nerve and optic nerve sheath were not clear enough. Previous data have indicated that the most common structural lesions include lacrimal gland (62-88\% of cases), orbital fat $(29-40 \%)$, EOM (19-25\%), and trigeminal nerve (10-39\%) [18, 19]. Other structures may involve eyelid, conjunctiva [20], optic nerve [21, 22] and mass compression. Song et al have found that IgG4-ROD mass lesions possess homogenous internal architecture, well-defined margins, contrast enhancement, and no destruction of orbit bone [23]. In this study, compression was the main cause of optic nerve damage, especially the mass compression, and most EOMs belonged to the pseudotumor mass compression, because the hypertrophy of the EOMs is a characteristic of pseudotumor mass. Previous data have suggested that IgG4-RD has a particular tropism for cranial nerves [24-26]. In addition, it has been reported that IONE can oppress the optic nerve at the

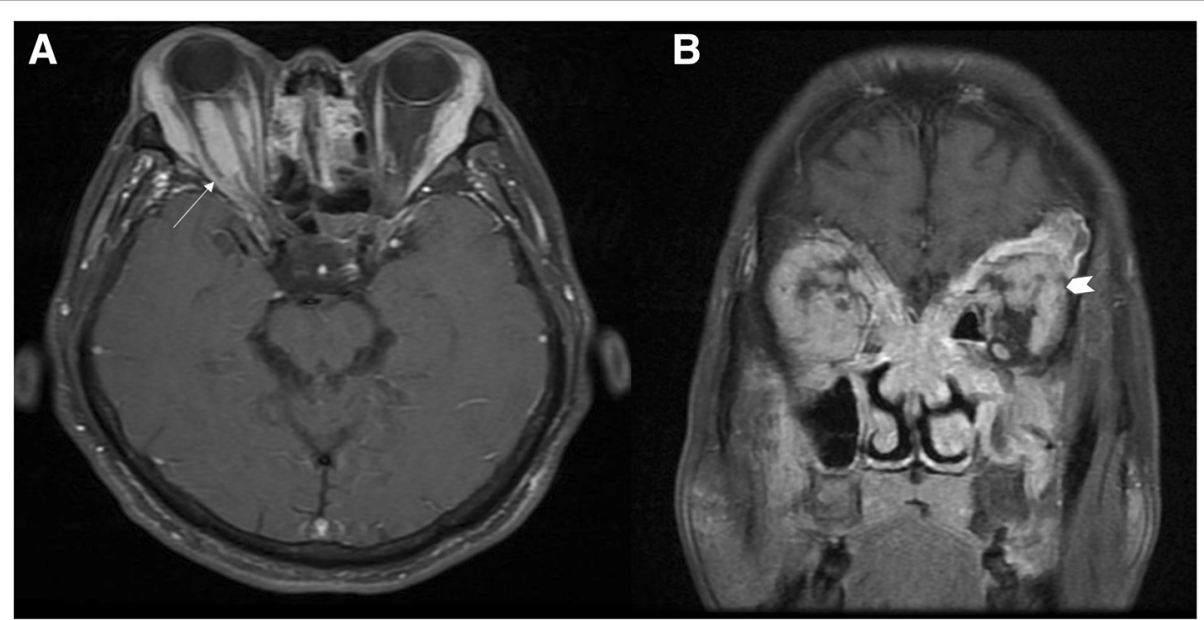

Fig. 5 Optic nerve was compressed by pseudotumor masses. Axial and coronal contrast enhanced T1-weighted MRI showing pseudotumor mass circles of optic nerve, was compressed by pseudotumor masses (white arrow) (a). Extraocular muscles appeared enlarged as masses and optic nerves were oppressed (white arrowhead) (b) 


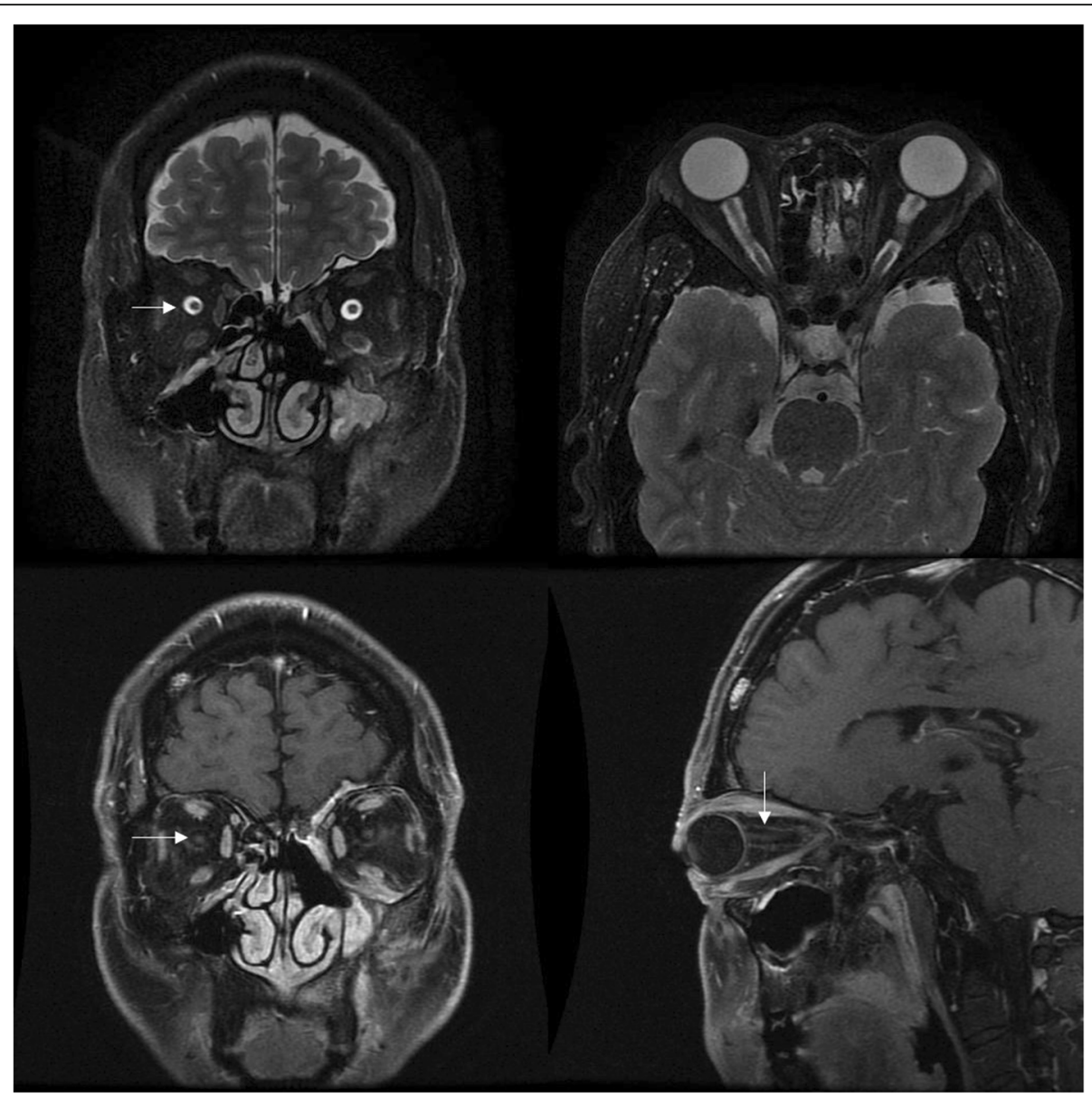

Fig. 6 Optic neuropathy with the reason of optic nerve sheath inflammation. Optic neuritis is characterized by diffuse thickening of the optic nerve sheath, blurred margin and enhanced. Optic neuritis was accompanied by orbital liposome inflammation, possibly due to the inflammation of orbital liposome involved in the optic nerve sheath

orbital apex [27]. However, in this study, we observed no IONE oppression related cases, while most oppressions were caused by pseudo tumor. In the current study, all the cases showed bilateral involvement, while $80.77 \%$ (21/26 cases) showed extraophthalmic involvement. Moreover, a Korean study group found that bilateral involvement, longer duration of symptom and higher IgG4 levels are all significant risk factors for extraophthalmic involvement in patients with IgG4-ROD [28]. IgG4-ROD with optic neuropathy involvement was more likely combined with extraophthalmic disorder. Accordingly, optic nerve involvement might be considered as another risk factor for extraophthalmic involvement.

IONE is the main sign for differentiating optic nerve sheath inflammation of IgG4-ROD from other orbital diseases. The orbital region is the first most frequent site of the head and neck region associated with the suffering from IgG4-RD. [3, 4] The main pathogenesis of optic neuropathy is compression; however, optic nerve sheath inflammation is also commonly observed. In addition, optic nerve sheath inflammation is often involved in unidiopathic orbital inflammation diseases [6]. IgG4-ROD diagnoses may be useful for the adequate management and appropriate care of their patients. In the current study, matched patients with non-IgG4 ROD were compared with IgG-4 ROD patients, IONE was found to be a specific sign of IgG4-ROD. A previous research has identified an association between the enlargement of infraorbital branch of the trigeminal nerve and the IgG4-ROD $[15,19,29]$. In previously mentioned study on IgG4-ROD that included European and Japanese study group, IONE was considered as the specific radiological feature $[5,30]$. In addition, according to radiological reviews on IgG4-ROD, trigeminal nerve enlargement occured in $24 \%$ (4/17 cases) [9], $29 \%$ (20/68 cases) [29], 39\% (25/65 cases) [19], and 50\% $(8 / 16$ cases) of cases [15]. In addition, one study $(n=$ 14) found that $50 \%$ of patients with IONE had IgG4ROD [26]. 


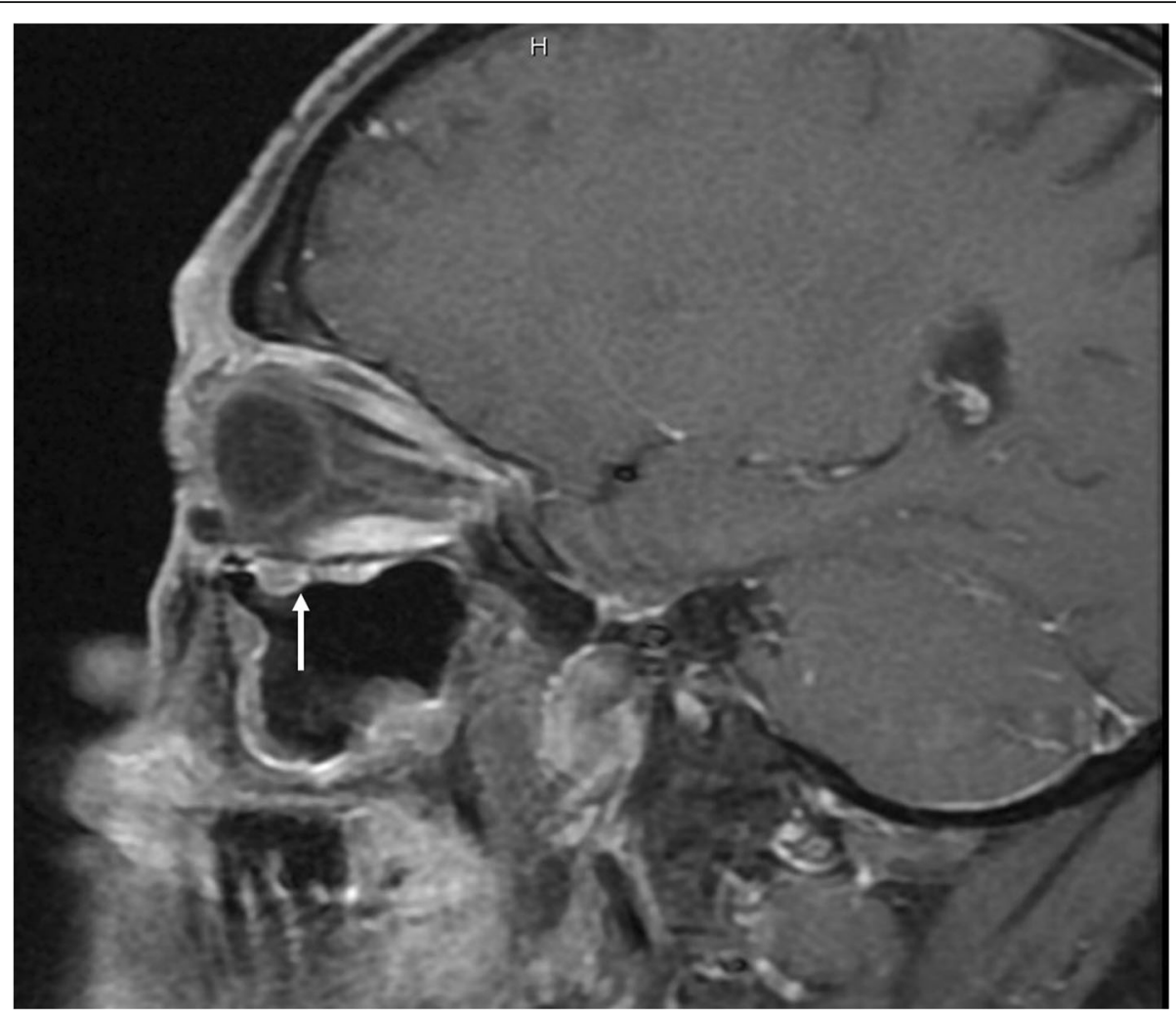

Fig. 7 Optic nerve sheath enhancement in patients accompanied with IONE. Sagittal contrast enhanced T1-weighted MRI shows enhancement of optic nerve sheath with IONE (white arrowhead)

Our study has few limitations. This is a study with retrospective design that was conducted only in two centers, thus non-controlled investigation and treatment protocol were considered as limitations. In addition, only 26 cases were included in this study, which means that future studies should include bigger sample size.

\section{Conclusion}

The most common imaging feature of the optic nerve for IgG4 is compression, while inflammation of the optic nerve sheath is the main reason affecting the visual function. Enhancement of optic nerve sheath is very likely to be combined with dacryoadenitis and infraorbital nerve enlargement. In addition, IONE and infraorbital nerve downwards resulted as important indications for the diagnosis of IgG4-ROD.

\section{Abbreviations}

EOMs: Extraocular muscles; IgG4-ROD: Immunoglobulin G4 (IgG4)-related ophthalmic diseases; IONE: Infraorbital nerve enlargement; MRI: Magnetic resonance imaging; VA: Visual acuity

\section{Acknowledgements}

Not applicable

\section{Authors' contributions}

$H Y L$ was involved in the conception and design of the study. J $L$ and ZC W reviewed the orbit MRI images. $J L$ and $Y Z$ contributed to the acquisition, analysis and interpretation of data as well as drafting the manuscript and revising it critically. $\mathrm{HZ}$ and $\mathrm{HY} \mathrm{L}$ diagnosed the disease, $\mathrm{L} W$ was involved in the conception and design of the study. ZC W has also provided final approval of the version to be published. All authors have given final approval of the version to be published. All authors have agreed to be accountable for all aspects of the work in ensuring that questions related to the accuracy or integrity of any part of the work are appropriately investigated and resolved.

\section{Funding}

This study was supported by the Beijing Natural Science Foundation (No. 7162048); In the role of analysis, and interpretation of data. Beijing Municipal Science \& Technology Commission (NO. Z171100001017163); In the role of collection data.

Start Funding of Beijing Friendship Hospital (No. yyqdkt2016-8); In the role of design of the study, collection, analysis data and writing manuscript.

\section{Availability of data and materials}

All data are contained within the manuscript.

Ethics approval and consent to participate

All the performed procedures involving human participants were in accordance with the ethical standards of the local ethics committee of Bioethics Committee of Beijing Friendship Hospital, Capital Medical University and with the 2013 Helsinki Declaration and the guideline of the International Conference on Harmonization of Good Clinical Practice and the applicable Chinese laws. In addition, all participants provided written or oral informed consent. 


\section{Consent for publication}

Not Applicable.

\section{Competing interests}

The authors declare that they have no competing interests.

\section{Author details}

'Department of Ophthalmology, Beijing Friendship Hospital, Capital Medica University, No. 95 Yong'an Road, Xicheng District, Beijing 100050, People's Republic of China. ${ }^{2}$ Department of Radiology, Beijing Friendship Hospital, Capital Medical University, Beijing, China. ${ }^{3}$ Department of Ophthalmology, PLA Army General Hospital, No.5, nanmencang, Dongsishitiao, dongcheng district, Beijing 100000, China. ${ }^{4}$ Department of Rheumatology, Beijing Friendship Hospital, Capital Medical University, Beijing, China. ${ }^{5}$ Department of Ophthalmology, PLA General Hospital, No.28, fuxing road, haidian district, Beijing, China100080.

Received: 8 December 2018 Accepted: 31 May 2019

Published online: 18 June 2019

\section{References}

1. Yamamoto M, Takahashi H, Ohara M, et al. A new conceptualization for Mikulicz's disease as an IgG4-related plasmacytic disease [J]. Mod Rheumatol. 2006:16(6):335-40.

2. Kawano M, Yamada K, Kakuchi Y, et al. A case of immunoglobulin G4related chronic sclerosing sialadenitis and dacryoadenitis associated with tuberculosis [J]. Mod Rheumatol. 2009;19(1):87-90.

3. Mulholland GB, Jeffery CC, Satija P, et al. Immunoglobulin G4-related diseases in the head and neck: a systematic review [J]. J Otolaryngol Head Neck Surg. 2015:44:24.

4. Andrew NH, Sladden N, Kearney DJ, et al. An analysis of IgG4-related disease (lgG4-RD) among idiopathic orbital inflammations and benign lymphoid hyperplasias using two consensus-based diagnostic criteria for IgG4-RD [J]. Br J Ophthalmol. 2015;99(3):376-81.

5. Soussan JB, Deschamps R, Sadik JC, et al. Infraorbital nerve involvement on magnetic resonance imaging in European patients with IgG4-related ophthalmic disease: a specific sign [J]. Eur Radiol. 2017:27(4):1335-43.

6. Tiegs-Heiden CA, Eckel LJ, Hunt CH, et al. Immunoglobulin G4-related disease of the orbit: imaging features in 27 patients [J]. AJNR Am J Neuroradiol. 2014;35(7):1393-7.

7. Woo YJ, Kim JW, Yoon JS. Clinical implications of serum lgG4 levels in patients with IgG4-related ophthalmic disease [J]. Br J Ophthalmol. 2017; 101(3):256-60.

8. Katsura M, Mori H, Kunimatsu A, et al. Radiological features of IgG4-related disease in the head, neck, and brain [J]. Neuroradiology. 2012;54(8):873-82.

9. Yu WK, Kao SC, Yang CF, et al. Ocular adnexal lgG4-related disease: clinical features, outcome, and factors associated with response to systemic steroids [J]. Jpn J Ophthalmol. 2015;59(1):8-13.

10. Umehara H, Okazaki K, Masaki Y, et al. Comprehensive diagnostic criteria for IgG4-related disease (IgG4-RD), 2011[J]. Mod Rheumatol. 2012;22(1):21-30.

11. Ozgen A, Ariyurek M. Normative measurements of orbital structures using CT [J]. AJR Am J Roentgenol. 1998;170(4):1093-6.

12. Ozgen A, Aydingoz U. Normative measurements of orbital structures using MRI [J]. J Comput Assist Tomogr. 2000;24(3):493-6.

13. Bateman AC, Deheragoda MG. IgG4-related systemic sclerosing disease - an emerging and under-diagnosed condition [J]. Histopathology. 2009;55(4): 373-83.

14. Kubota T, Moritani S. Orbital IgG4-related disease: clinical features and diagnosis [J]. ISRN Rheumatol. 2012:2012:412896.

15. Ohshima K, Sogabe Y, Sato Y. The usefulness of infraorbital nerve enlargement on MRI imaging in clinical diagnosis of lgG4-related orbital disease [J]. Jpn J Ophthalmol. 2012;56(4):380-2.

16. Ginat DT, Freitag SK, Kieff D, et al. Radiographic patterns of orbital involvement in IgG4-related disease [J]. Ophthal Plast Reconstr Surg. 2013; 29(4):261-6

17. Origuchi $\mathrm{T}$, Yano $\mathrm{H}$, Nakamura $\mathrm{H}$, et al. Three cases of IgG4-related orbital inflammation presented as unilateral pseudotumor and review of the literature [J]. Rheumatol Int. 2013:33(11):2931-6.

18. Wallace ZS, Deshpande V, Stone JH. Ophthalmic manifestations of IgG4related disease: single-center experience and literature review [J]. Semin Arthritis Rheum. 2014;43(6):806-17.
19. Sogabe Y, Ohshima K, Azumi A, et al. Location and frequency of lesions in patients with lgG4-related ophthalmic diseases [J]. Graefes Arch Clin Exp Ophthalmol. 2014;252(3):531-8.

20. Paulus YM, Cockerham KP, Cockerham GC, et al. IgG4-positive sclerosing orbital inflammation involving the conjunctiva: a case report [J]. Ocul Immunol Inflamm. 2012;20(5):375-7.

21. Deschamps R, Deschamps L, Depaz R, et al. High prevalence of IgG4-related lymphoplasmacytic infiltrative disorder in 25 patients with orbital inflammation: a retrospective case series [J]. Br J Ophthalmol. 2013;97(8): 999-1004.

22. Caputo C, Bazargan A, McKelvie PA, et al. Hypophysitis due to IgG4-related disease responding to treatment with azathioprine: an alternative to corticosteroid therapy [J]. Pituitary. 2014;17(3):251-6.

23. Song YS, Choung HK, Park SW, et al. Ocular adnexal IgG4-related disease: CT and MRI findings [J]. Br J Ophthalmol. 2013:97(4):412-8.

24. Wallace ZS, Khosroshahi A, Jakobiec FA, et al. IgG4-related systemic disease as a cause of "idiopathic" orbital inflammation, including orbital myositis, and trigeminal nerve involvement [J]. Surv Ophthalmol. 2012;57(1):26-33.

25. Fathala A. Multimodalities imaging of immunoglobulin 4-related cardiovascular disorders. Curr Cardiol Rev. 2019;15(3):224-9.

26. Hardy TG, McNab AA, Rose GE. Enlargement of the infraorbital nerve: an important sign associated with orbital reactive lymphoid hyperplasia or immunoglobulin g4-related disease [J]. Ophthalmology. 2014;121(6):1297-303.

27. Kashii S. IgG4-related disease: a neuro-ophthalmological perspective [J]. J Neuroophthalmol. 2014;34(4):400-7.

28. Park J, Lee MJ, Kim N, et al. Risk factors for extraophthalmic involvement and treatment outcomes in patients with IgG4-related ophthalmic disease [J]. Br J Ophthalmol. 2018 Jun;102(6):736-41.

29. Takano K, Yajima R, Seki N, et al. A study of infraorbital nerve swelling associated with immunoglobulin G4 Mikulicz's disease [J]. Mod Rheumatol. 2014;24(5):798-801.

30. Goto H, Takahira M, Azumi A. Diagnostic criteria for IgG4-related ophthalmic disease [J]. Jpn J Ophthalmol. 2015:59(1):1-7.

\section{Publisher's Note}

Springer Nature remains neutral with regard to jurisdictional claims in published maps and institutional affiliations.

Ready to submit your research? Choose BMC and benefit from:

- fast, convenient online submission

- thorough peer review by experienced researchers in your field

- rapid publication on acceptance

- support for research data, including large and complex data types

- gold Open Access which fosters wider collaboration and increased citations

- maximum visibility for your research: over $100 \mathrm{M}$ website views per year

At $\mathrm{BMC}$, research is always in progress.

Learn more biomedcentral.com/submissions 